\title{
Genetic variants in the exon region of versican predict survival of patients with resected early- stage hepatitis B virus-associated hepatocellular carcinoma
}

This article was published in the following Dove Press journal:

Cancer Management and Research

\author{
Xiaoguang Liu* \\ Chuangye Han* \\ Xiwen Liao \\ Long Yu \\ Guangzhi Zhu \\ $\mathrm{Hao} \mathrm{Su}$ \\ Wei Qin \\ Sicong Lu \\ Xinping Ye \\ Tao Peng
}

Department of Hepatobiliary Surgery, The First Affiliated Hospital of

Guangxi Medical University, Nanning,

Guangxi Zhuang Autonomous Region,

China

*These authors contributed equally to this work
Correspondence: Tao Peng

Department of Hepatobiliary Surgery,

The First Affiliated Hospital of Guangxi

Medical University, Nanning 53002I,

Guangxi Zhuang Autonomous Region,

People's Republic of China

Tel +86 77I 5356528

Fax +86 77| 535003 I

Email pengtaogmu@।63.com
Background: The upregulated expression of versican (VCAN) promotes the proliferation, invasion, and metastasis of various types of human cancer cells, including hepatocellular carcinoma (HCC) cells.

Patients and methods: In this study, genetic variants in the exon region of $V C A N$ were genotyped by DNA sequencing. Prognostic values of $V C A N$ exon single nucleotide polymorphisms (SNPs) were assessed by Kaplan-Meier with the log-rank test, and uni- and multivariate Cox proportional hazard regression model.

Results: A total of 111 patients with resected hepatitis B virus-associated early-stage HCC were collected for genotyping $V C A N$ exon SNPs using Sanger DNA sequencing. Haplotype analysis was performed using Haploview 4.2. Survival data were analyzed using Kaplan-Meier curves and Cox proportional hazards regression analyses. The rs2652098, rs309559, rs188703, rs160278, and rs160277 SNPs were significantly associated with overall patient survival $(p<0.001, p=0.012$, $p=0.010, p=0.007$, and $p=0.007$, respectively). Patients carrying the TAGTG haplotype had a poorer prognosis than those with the most common CGAAT haplotype, after adjusting for tumor size, tumor capsule, and regional invasion (adjusted hazard ratio $[\mathrm{HR}]=2.06,95 \% \mathrm{CI}$ : $1.27-3.34, p=0.003$ ). Meanwhile, patients with the TAGTG haplotype and a larger tumor size or an incomplete tumor capsule had an increased risk of death, compared with the others (adjusted $\mathrm{HR}=3.00,95 \% \mathrm{CI}: 1.67-5.36, p<0.001$; and adjusted $\mathrm{HR}=1.99,95 \% \mathrm{CI}=1.12-3.55, p=0.02$, respectively). The online database mining analysis showed that upregulated VCAN expression in HCC tissues was associated with a poor overall survival of $148 \mathrm{HCC}$ patients.

Conclusion: Genetic variants in the exon region of $V C A N$ were associated with overall survival in patients with resected early-stage hepatitis B virus-associated HCC, and may be a potential prognostic biomarker.

Keywords: versican, single nucleotide polymorphism, hepatitis B virus, hepatocellular carcinoma, survival

\section{Introduction}

Liver cancer is a lethal disease, and in 2012 alone, there were an estimated 782,500 new liver cancer cases and 745,500 liver cancer-related deaths occurred in the world. ${ }^{1}$ To date, $70 \%-90 \%$ of primary liver cancers are hepatocellular carcinoma (HCC). The highest incidence of HCC is in Asia, and half of HCC cases occur in China. ${ }^{2-4}$ The treatment options for HCC patients include hepatic resection, orthotopic liver transplantation, loco-regional chemotherapy, radiation therapy, and systemic or targeting therapy. ${ }^{5}$ Among them, hepatic resection is the first-line curative treatment, but it is only 
available for early-stage HCC patients; and the vast majority of HCC patients are not suitable for surgery due to hepatitis $\mathrm{B}$ virus (HBV)- or hepatitis $\mathrm{C}$ virus ( $\mathrm{HCV}$ )-induced liver cirrhosis or poor liver function. ${ }^{6}$ Even after a hepatectomy, there is a $50 \%-60 \%$ chance of tumor recurrence, leading to a poor outcome for patients with HBV-associated HCC. ${ }^{6}$ Overall, a large tumor size, multiple tumor lesions, poor tumor cell differentiation, vascular invasion, and degree of inflammation have been associated with a poor HCC prognosis. ${ }^{7}$ Thus, identification and evaluation of novel biomarkers could help medical oncologists to select patients for more aggressive or targeted therapy in order to improve the outcome of $\mathrm{HCC}$ after surgery.

Versican (VCAN) is a large extracellular matrix proteoglycan that is expressed in majority human tissues. $V C A N$ is localized on human chromosome $5 q 14.3$, covering a genomic sequence of $109.4 \mathrm{~kb} .{ }^{8}$ VCAN cDNA contains 15 exons and codes a protein with a molecular mass of $>1000 \mathrm{kDa}$, which belongs to the aggrecan/versican proteoglycan family. VCAN protein functions to regulate cell adhesion, proliferation, migration, and angiogenesis; and it plays a central role in tissue morphogenesis and maintenance. ${ }^{9-14}$ Altered VCAN expression is closely related to proliferation, invasion, and metastasis in various types of human cancer cells, including HCC, and upregulated VCAN expression has been associated with a poor prognosis of cervical cancer patients. ${ }^{15}$ To date, VCAN has 4 splice variants, that is, V0, V1, V2, and V3, each of which appears to have distinct biological functions. ${ }^{16-18}$ Previous studies also have shown that $V C A N$ genetic polymorphisms are associated with the development of gastric cancer and that altered VCAN expression is associated with outcomes in ovarian, breast, prostate, and gastric cancer. ${ }^{19-24}$ Versican V1 has been shown to promote HCC metastasis, and its expression has been associated with the prognosis of $\mathrm{HCC}$ patients after curative resection. ${ }^{9}$ VCAN contains 2 globular domains, that is, the G1 domain (N-terminal; G1-D), possessing the hyaluronan-binding property, and the G3 domain (C-terminal; G3-D), consisting of epidermal growth factor repeats. ${ }^{25}$ Another study has reported that the presence of the rs 188703 variant allele $\mathrm{A}$ and the rs 160277 variant allele $\mathrm{T}$ is a susceptibility genotype for gastric cancer, while the rs309559 variant allele $G$ and the rs 160278 variant allele $A$ are also susceptibility factors in the development of gastric cancer. ${ }^{24}$ Therefore, in this study, we assessed 5 different polymorphisms in the exon region of $V C A N$ and then associated them with the prognosis of resected early-stage $\mathrm{HBV}$-associated $\mathrm{HCC}$ patients. These genetic polymorphisms in the $V C A N$ exon region lead to changes in the VCAN protein amino acids; thus, we also performed an online database mining analysis to associate VCAN expression in HCC tissues with HCC patient survival. This study will provide insightful information regarding these $V C A N$ single-nucleotide polymorphisms (SNPs) as a novel prognostic biomarker for HCC patients.

\section{Patients and methods \\ Study population}

The study protocol was approved by the Ethics Committee of the First Affiliated Hospital of Guangxi Medical University (approval no. 2015KY-E-032). The written informed consents were obtained from all the participants enrolled in the study. From January 2005 to September 2013, we collected 111 patients with early-stage HBV-associated HCC among 1150 patients diagnosed with HBV-related HCC from The First Affiliated Hospital, Guangxi Medical University (Nanning, China). All of these 111 patients underwent a hepatectomy and had a confirmed HCC diagnosis based on histopathology. Tumor differentiation was assessed according to the Edmondson-Steiner grading system. ${ }^{26}$ Grade I was considered well-differentiated $\mathrm{HCC}$, grades II and III were considered moderately differentiated $\mathrm{HCC}$, and grade IV was considered poorly differentiated HCC. The inclusion criteria for patient enrollment into this study were as follows: 1) patients who tested positive for hepatitis B surface antigen, 2) patients with Barcelona Clinic Liver Cancer (BCLC) stage A, ${ }^{27,28}$ and 3) patients with Child-Pugh class A liver disease. The exclusion criteria were as follows: 1) patients with $\mathrm{HCV}$ infection and 2) patients with other cancers. The clinicopathological data were collected from the patients' medical records and are summarized in Table 1.

In this study, radical hepatic resection was defined as the complete removal of the tumor lesion with an incisional margin $\geq 2 \mathrm{~cm}$ and without a visible tumor thrombus in the hepatic vein or in the extrahepatic lymph nodes. These patients were followed up in our outpatient clinic or through a telephone interview every 3 months, and the last followup was conducted on September 1, 2014. This study was approved by the Ethics Committee of The First Affiliated Hospital of Guangxi Medical University (Nanning, China).

\section{SNP selection, DNA extraction, and genotyping}

The SNPs in the $V C A N$ exon region that are responsible for changes in amino acids were selected with a minor allele frequency $>5 \%$ in the Han Chinese population from the 1000 Genomes Project (March 2012, build GRCh37/hg19). Five SNPs in the VCAN gene exon region (rs2652098, rs309559, 
Table I Clinical features of patients with resected early-stage HBV-associated HCC

\begin{tabular}{|c|c|c|c|c|}
\hline Variables & $n=I I I$ & MST (months) & HR (95\% CI) & $p$-value \\
\hline Age (years) & & & & 0.846 \\
\hline$\leq 45$ & 63 & 47 & 1 & \\
\hline$>45$ & 48 & 45 & $1.05(0.65-1.68)$ & \\
\hline Gender & & & & 0.61 \\
\hline Male & 97 & 45 & I & \\
\hline Female & 14 & 51 & $0.83(0.40-1.73)$ & \\
\hline Smoking status & & & & 0.49 \\
\hline None & 76 & 57 & 1 & \\
\hline Ever & 35 & 42 & $1.19(0.72-1.97)$ & \\
\hline Drinking status & & & & 0.44 \\
\hline None & 64 & 42 & I & \\
\hline Ever & 47 & 82 & $0.83(0.5 \mathrm{I}-1.34)$ & \\
\hline $\operatorname{AFP}(\mathrm{ng} / \mathrm{mL})^{\mathrm{a}}$ & & & & 0.64 \\
\hline$\leq 400$ & 58 & 57 & 1 & \\
\hline$>400$ & 47 & 41 & $1.12(0.69-1.83)$ & \\
\hline Tumor size $(\mathrm{cm})$ & & & & 0.020 \\
\hline$\leq 5$ & 36 & 80 & 1 & \\
\hline$>5$ & 75 & 42 & $1.94(1.10-3.45)$ & \\
\hline No. of tumors & & & & 0.92 \\
\hline Single $(n=1)$ & 106 & 45 & I & \\
\hline Multiple $(n>1)$ & 5 & 65 & $0.94(0.30-3.00)$ & \\
\hline Tumor capsule & & & & 0.024 \\
\hline Complete & 59 & 88 & I & \\
\hline Incomplete & 52 & 38 & $1.72(1.07-2.78)$ & \\
\hline Regional invasion & & & & $<0.001$ \\
\hline Absent & 101 & 74 & I & \\
\hline Present & 10 & 11 & $3.68(1.90-7.13)$ & \\
\hline Tumor differentiation ${ }^{\mathrm{b}}$ & & & & 0.64 \\
\hline Well & 9 & 79 & I & \\
\hline Moderately & 74 & 42 & $1.48(0.59-3.72)$ & \\
\hline Poorly & 2 & 40 & $0.96(0.11-8.25)$ & \\
\hline Cirrhosis & & & & 0.63 \\
\hline Absent & 17 & 82 & I & \\
\hline Present & 94 & 45 & $1.18(0.60-2.31)$ & \\
\hline Radical liver resection ${ }^{c}$ & & & & 0.69 \\
\hline Yes & 70 & 45 & 1 & \\
\hline No & 38 & 38 & $1.10(0.67-1.81)$ & \\
\hline Adjuvant antiviral treatment & & & & 0.29 \\
\hline No & 95 & 65 & I & \\
\hline Yes & 16 & 25 & $1.41(0.74-2.69)$ & \\
\hline Adjuvant TACE & & & & 0.12 \\
\hline No & 39 & 101 & 1 & \\
\hline Yes & 72 & 42 & $1.51(0.89-2.57)$ & \\
\hline
\end{tabular}

Notes: aAP level information are unavailable for 6 patients. ${ }^{b}$ Pathological diagnosis information are unavailable for 16 patients. ${ }^{\mathrm{C}}$ Radical hepatic resection information are unavailable for 3 patients. These $P$-values $<0.05$ were highlighted in bold font.

Abbreviations: AFP, alpha-fetoprotein; HBV-associated HCC, hepatitis B virus-associated hepatocellular carcinoma; HR, hazard ratio; MST, median survival time; TACE, transarterial chemoembolization.

rs188703, rs160278, and rs160277) were identified and assessed in this study.

Tissue samples from HCC lesions were obtained after surgical resection, immediately snap-frozen, and stored at $-80^{\circ} \mathrm{C}$ until needed for DNA extraction. Genomic DNA from the HCC tissue samples was extracted using a TIANamp Genomic DNA Kit (Tiangen Biotech [Beijing] Co., Ltd., Beijing, China), according to the manufacturer's protocol.
VCAN genetic variations were genotyped by Sanger DNA sequencing on an ABI Prism 3100 (Applied Biosystems, Shanghai Sangon Biological Engineering Technology \& Services Co., Ltd., Shanghai, China) after polymerase chain reaction amplification using different primers (Table 2). Ten samples were randomly selected for repeated polymerase chain reaction, the DNA sequences were used to validate the genotyping data, and the results showed $100 \%$ concordance. 
Table 2 Primer sequences and HWE of VCAN exon polymorphisms

\begin{tabular}{|c|c|c|c|c|}
\hline SNPs & Primer sequences & Allele $^{a}$ & Function & HWE $p$-value \\
\hline \multirow[t]{2}{*}{ rs2652098 } & 5'-AGAAGCTGCTACCTTGCCATGA-3' & $\mathrm{C}>\mathrm{T}$ & Ser300Leu & 0.035 \\
\hline & 5'-TGTTTCCATTCCGTGCACACAC-3' & & & \\
\hline \multirow[t]{2}{*}{ rs309559 } & 5'-AGTTACATGGAAGCCTGAGACTTAC-3' & $A>G$ & Lys1516Arg & 0.069 \\
\hline & 5'-TTGGTCAATGGCAATCTCTCCTG-3' & & & \\
\hline \multirow[t]{2}{*}{ rs 188703} & 5'-AGTCTGAAAGGGAAATGACAGATTCTAC-3' & $\mathrm{G}>\mathrm{A}$ & Argl826His & 0.69 \\
\hline & 5'-AGCTACTTCCTTTTCGGCTTGAA-3' & & & \\
\hline \multirow[t]{2}{*}{ rs 160278} & 5'-CCAACAATTCAAGAGTCAGATACTGAGC-3' & $A>T$ & Phe230ITyr & 0.15 \\
\hline & 5'-CCCACTACCTTCAAAGATGTCTGTT-3' & & & \\
\hline \multirow[t]{2}{*}{ rsI60277 } & 5'-ACCTTCAGAAGATGATGGTAAACCT-3' & $\mathrm{G}>\mathrm{T}$ & Asp2937Tyr & 0.73 \\
\hline & 5'-TCATTAGAAGTCTCCAGAAGGGAAA-3' & & & \\
\hline
\end{tabular}

Note: ${ }^{a}$ Common allele $>$ the variant allele.

Abbreviations: HWE, Hardy-Weinberg equilibrium; SNP, single-nucleotide polymorphism; VCAN, versican.

\section{Online mining of VCAN gene expression data}

To associate and confirm $V C A N$ expression with HCC patient survival, we searched the online database (GSE14520 [https:// www.ncbi.nlm.nih.gov/geo/query/acc.cgi?acc=GSE14520] in the National Center for Biotechnology Information (NCBI) Gene Expression Omnibus (GEO) database) and obtained a cohort of 148 early-stage HBV-associated HCC patients (BCLC stage A) from Zhongshan Hospital, Fudan University (Shanghai, China). ${ }^{29,30}$ We used this cohort of patients and data as a validated set. These patients were similar to our cohort, with the same inclusion and exclusion criteria.

\section{Statistical analysis}

Hardy-Weinberg equilibrium for each SNP was estimated using a goodness-of-fit $\chi^{2}$-test with one degree of freedom. The linkage disequilibrium between SNPs of the $V C A N$ gene and the haplotype block were conducted using Gabriel's algorithm in Haploview version 4.2. ${ }^{31}$ For all of these 5 SNPs, 1 reasonable genetic model (dominant, additive, or recessive models) was used. Overall survival (OS) was defined from the date of the hepatectomy to death or the last follow-up (September 1, 2014). The univariate analysis between clinical features and OS was compared using the log-rank test, and those with $p<0.1$ were entered into the Cox proportional hazard regression model. A $p$-value $<0.05$ was defined as statistically significant. All statistical analyses were performed using SPSS 16.0 (SPSS, Inc., Chicago, IL, USA).

\section{Results}

\section{Patient characteristics and clinical predictors}

In this study, we collected 111 cases of HBV-related HCC with BCLC stage A and Child-Pugh class A liver disease.
Their clinicopathological characteristics are summarized in Table 1. In brief, the median patient age was 45 years old, there were $87.4 \%$ male patients, $67.6 \%$ of patients had a maximum tumor diameter $\geq 10 \mathrm{~cm}$, and $84.7 \%$ of patients had liver cirrhosis. Moreover, $52.3 \%$ of patients had a low serum alpha-fetoprotein (AFP) level ( $\leq 400 \mathrm{ng} / \mathrm{mL}$ ), $31.5 \%$ of patients had ever smoked tobacco, and $42.3 \%$ of patients drank alcohol. Seventy patients $(63.1 \%)$ underwent a radical hepatic resection, 72 (64.9\%) patients received a hepatic resection and adjuvant transarterial chemoembolization (TACE), and 95 (85.6\%) patients did not receive adjuvant antiviral treatment.

The median follow-up period was 47 months (range: 10-117 months). During the follow-up, 69 (62.2\%) patients had died, including $50(45.0 \%)$ patients who died due to HCC recurrence. Tumor size, tumor capsule, and regional invasion were all associated with the OS of these patients (tumor size, $p=0.020$; tumor capsule, $p=0.024$; tumor regional invasion, $p<0.001$ with the log-rank test). Patients with a maximum tumor diameter $\geq 5 \mathrm{~cm}$ had a $94.0 \%$ increased risk of death (hazard ratio $[\mathrm{HR}]=1.94$, 95\% CI: 1.10-3.45). Patients with an incomplete tumor capsule also had a higher risk of death $(\mathrm{HR}=1.72,95 \%$ CI: 1.07-2.78). Moreover, patients who were treated with postoperative adjuvant antiviral therapy or adjuvant TACE did not show a decreased risk of death (adjuvant antiviral treatment, $p=0.29$; adjuvant TACE, $p=0.12$ with the logrank test).

\section{Associations of SNPs with overall patient survival}

The Hardy-Weinberg equilibrium data for these 5 SNPs are listed in Table 2. All these 5 SNPs had 3 genotypes, and all 
of them were significantly associated with overall patient survival after adjusting for tumor size, tumor capsule, and regional invasion according to the Cox proportional hazard regression analyses (Table 3). The rs2652098 SNP was shown to be the most significantly associated with a shorter OS in patients who carried the T allele (CT/TT) than in those with the wild-type CC allele (adjusted HR $=2.42,95 \%$ CI: $1.49-3.94$, under the dominant genetic model, Table 3).

\section{Haplotype analysis of the association between SNPs and OS of HCC patients}

Haplotype analysis of the SNPs rs2652098, rs309559, rs188703, rs160278, and rs160277 identified 1 haplotype block (Figure 1A). Haplo4 TAGTG was associated with a shorter median survival time, compared with those with the most common genotype CGAAT (34.0 vs. 83.0 months, $p=0.01$ with the log-rank test; Figure 1B). After adjusting for tumor size, tumor capsule, and regional invasion, patients with haplotype TAGTG had a significantly increased risk of death, compared with those with the genotype CGAAT (adjusted HR $=2.06,95 \%$ CI: $1.27-3.34$, $p=0.003$; Table 4 ).

\section{Stratified analysis of the association of VCAN haplotypes with OS of HCC patients}

Next, we carried out further stratified analyses by age, gender, AFP level, tumor size, tumor capsule, radical hepatic resection, adjuvant TACE, and adjuvant antiviral treatment to evaluate associations between the haplotype block of VCAN and OS of patients with HBV-associated HCC (Figure 2). Haplo1, Haplo2, or Haplo3 had a significant protective effect in male patients (adjusted HR $=0.64,95 \%$ CI: $0.42-0.96$, Figure 2) and in patients with serum AFP levels $>400 \mathrm{ng} / \mathrm{mL}$ (adjusted HR $=0.35,95 \%$ CI: $0.20-0.63$, Figure 2 ) and adjuvant TACE (adjusted HR $=0.58,95 \%$ CI: 0.36-0.94). Haplo1, Haplo2, or Haplo3 also significantly decreased the death risk in patients without radical hepatic resection (adjusted HR $=0.41,95 \%$ CI: $0.20-0.83$, Figure 2 ) and adjuvant antiviral therapy (adjusted HR $=0.54,95 \%$ CI: 0.36-0.82, Figure 2).

\section{Combined effects of the VCAN haplotype as well as tumor size and capsule on OS of HCC patients}

The combined effects of the VCAN haplotype and clinical features (tumor size and capsule) on the prediction of OS of

Table 3 Association of VCAN exon region SNPs with survival of patients with resected early-stage HBV-associated HCC

\begin{tabular}{|c|c|c|c|c|c|}
\hline SNPs & Best fitting model & Death/total & Crude HR (95\% Cl) & Adjusted HR $(95 \% \mathrm{Cl})^{\mathrm{a}}$ & $\begin{array}{l}\text { p-value } \\
\text { (adjusted) }\end{array}$ \\
\hline \multirow[t]{4}{*}{ rs2652098 } & $\mathrm{CC}$ & $31 / 63$ & $\mathrm{I}$ & 1 & \\
\hline & CT & $35 / 42$ & $2.36(1.45-3.85)$ & $2.39(1.46-3.90)$ & 0.001 \\
\hline & $\mathrm{TT}$ & $\mathrm{I} / \mathrm{I}$ & $6.19(0.819-46.81)$ & $5.97(0.77-46.54)$ & 0.088 \\
\hline & Dominant & & $2.40(1.48-3.90)$ & $2.42(1.49-3.94)$ & $<0.001$ \\
\hline \multirow[t]{4}{*}{ rs309559 } & GG & $12 / 29$ & I & 1 & \\
\hline & GA & $29 / 44$ & $1.96(1.00-3.85)$ & $2.09(1.05-4.15)$ & 0.036 \\
\hline & AA & $25 / 34$ & $2.59(1.29-5.16)$ & $2.50(1.24-5.04)$ & 0.011 \\
\hline & Recessive & & $2.21(1.18-4.13)$ & $2.26(1.19-4.28)$ & 0.012 \\
\hline \multirow[t]{4}{*}{ rs 188703} & AA & $6 / 17$ & I & 1 & \\
\hline & AG & $31 / 50$ & $2.12(0.88-5.09)$ & $2.86(1.16-7.01)$ & 0.022 \\
\hline & GG & $31 / 43$ & 3.07 (1.27-7.38) & $3.37(1.38-8.22)$ & 0.008 \\
\hline & Additive & & $1.63(1.14-2.33)$ & $1.58(1.12-2.24)$ & 0.010 \\
\hline \multirow[t]{4}{*}{ rs 160278} & AA & $11 / 28$ & I & 1 & \\
\hline & AT & $31 / 47$ & $2.18(1.09-4.33)$ & $2.32(1.15-4.68)$ & 0.018 \\
\hline & TT & $25 / 34$ & $2.8 \mathrm{I}(1.38-5.73)$ & $2.70(1.30-5.49)$ & 0.008 \\
\hline & Dominant & & $2.42(1.26-4.62)$ & $2.47(1.28-4.77)$ & 0.007 \\
\hline \multirow[t]{4}{*}{ rs 160277} & TT & $5 / 16$ & 1 & 1 & \\
\hline & TG & $30 / 49$ & $2.40(0.93-6.20)$ & $3.35(1.26-8.89)$ & 0.015 \\
\hline & GG & $31 / 43$ & $3.45(1.34-8.90)$ & $3.87(1.47-10.13)$ & 0.006 \\
\hline & Recessive & & $2.83(1.14-7.06)$ & 3.61 (1.42-9.19) & 0.007 \\
\hline
\end{tabular}

Note: adjusted for tumor size, tumor capsule, regional invasion. These $P$-values $<0.05$ were highlighted in bold font.

Abbreviations: HBV-associated HCC, hepatitis B virus-associated hepatocellular carcinoma; HR, hazard ratio; SNP, single-nucleotide polymorphism; VCAN, versican. 
A

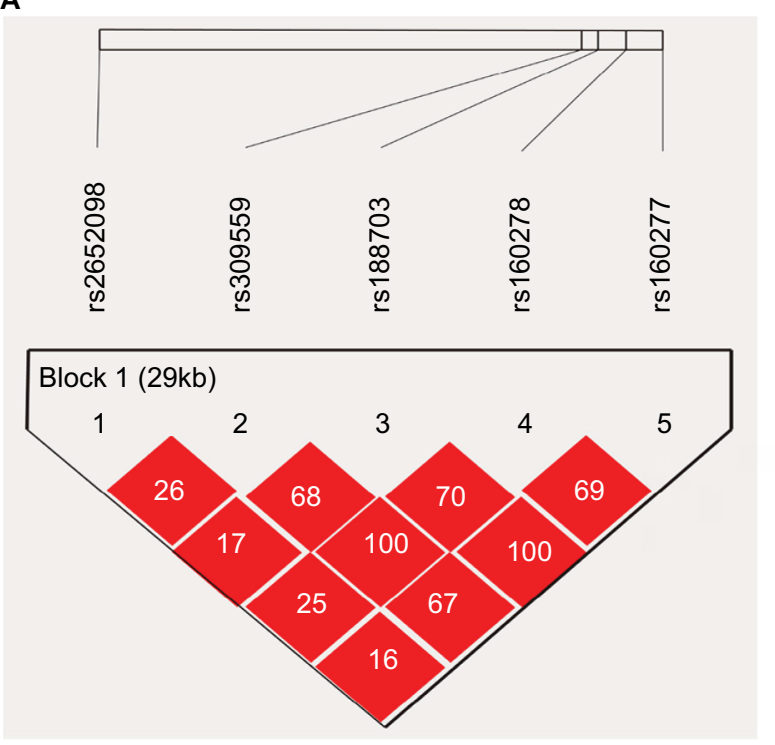

B

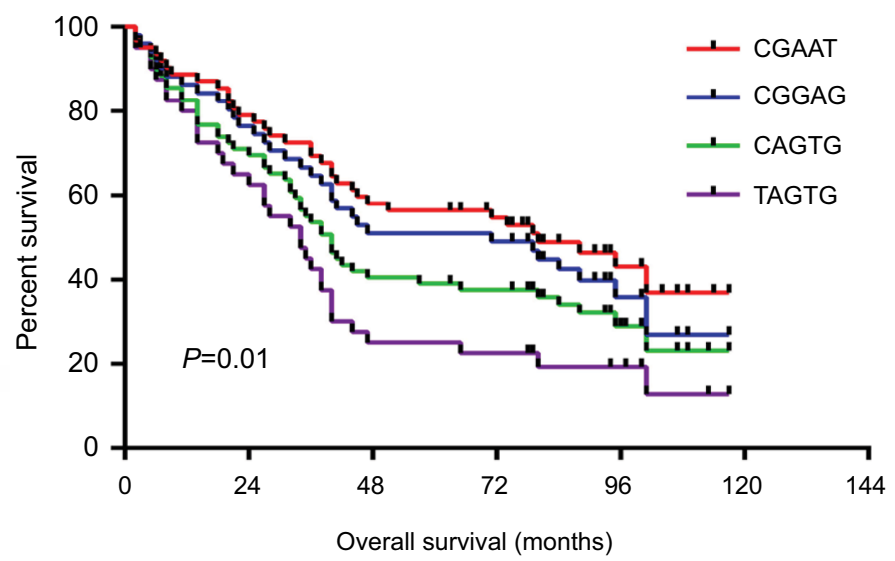

Figure I Association of VCAN SNP haplotypes with OS of patients with resected early-stage HBV-associated HCC.

Notes: (A) Patterns of LD plot for 5 selected VCAN SNPs. The value within each diamond represents the pairwise LD (correlation, measured as $r^{2}$ values) between these 5 SNPs defined by the upper left and the upper right sides of the diamond. One block was identified based on the LD patterns. (B) Kaplan-Meier curves of OS stratified by haplotype (TAGTG) and other haplotypes in patients with resected early-stage HBV-associated HCC.

Abbreviations: HBV-associated HCC, hepatitis B virus-associated hepatocellular carcinoma; LD, linkage disequilibrium; OS, overall survival; SNP, single nucleotide polymorphism; VCAN, versican.

Table 4 Associations between VCAN haplotypes and OS of patients with resected early-stage HBV-associated HCC

\begin{tabular}{llllll}
\hline Variables & Haplotypes & Death/total (2n) & MST (months) & Adjusted HR (95\% CI) $^{\mathbf{a}}$ & p-value (adjusted) $^{\mathbf{a}}$ \\
\hline Haplol & CGAAT & $34 / 62$ & 80 & $\mathrm{I}$ & \\
Haplo2 & CGGAG & $32 / 51$ & 71 & $1.26(0.78-2.04)$ & 0.35 \\
Haplo3 & CAGTG & $48 / 59$ & 40 & $1.47(0.94-2.28)$ & 0.091 \\
Haplo4 & TAGTG & $33 / 40$ & 34 & $2.06(1.27-3.34)$ & $\mathbf{0 . 0 0 3}$ \\
\hline
\end{tabular}

Notes: aAdjusted for tumor size, tumor capsule, and regional invasion. Haplotype analysis included rs2652098, rs309559, rs 188703, rs 160278, and rs I60277 SNPs. These $P$-values $<0.05$ were highlighted in bold font.

Abbreviations: HBV-associated HCC, hepatitis B virus- associated hepatocellular carcinoma; HR, hazard ratio; MST, median survival time; OS, overall survival; VCAN, versican.

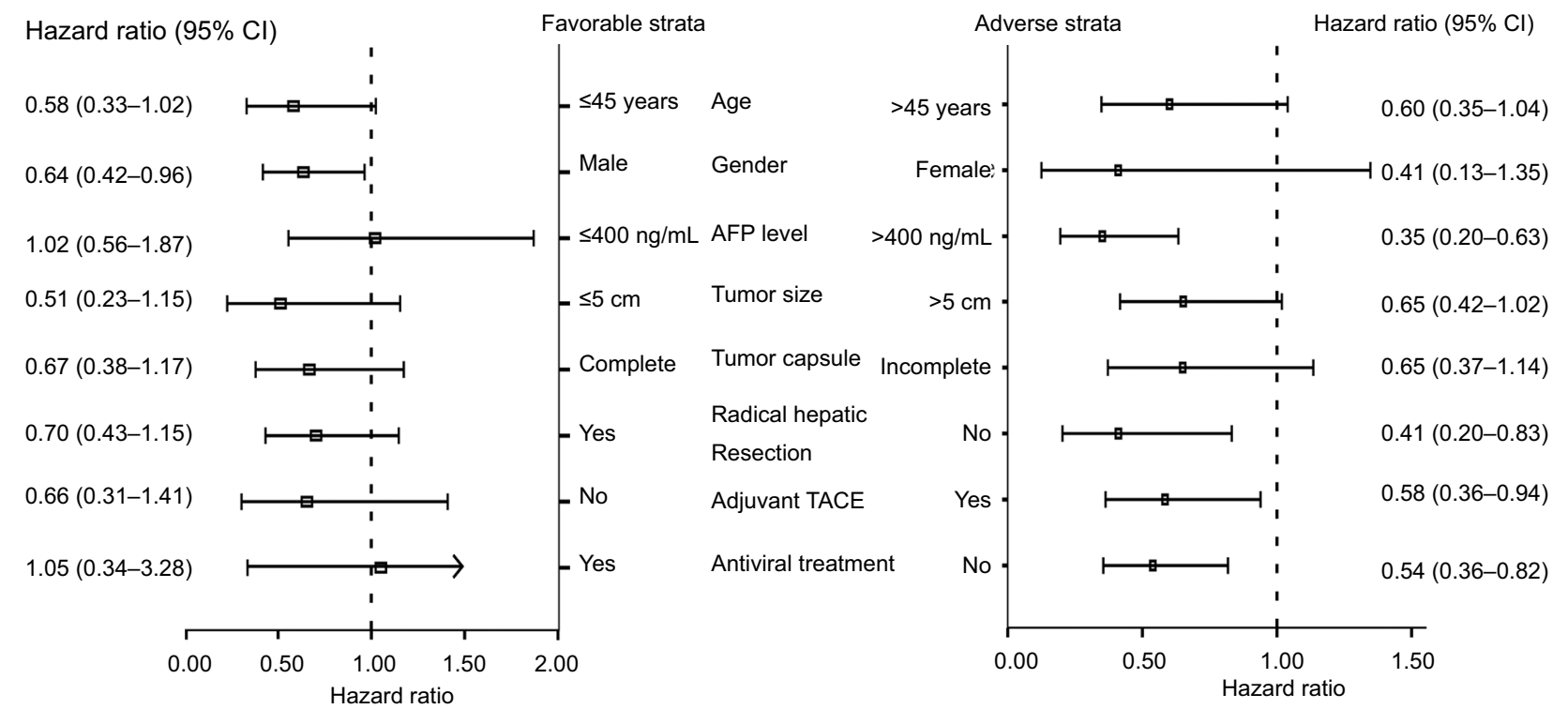

Figure 2 Stratified analysis of the association between VCAN haplotypes (TAGTG haplotype vs. other haplotypes) and OS of patients with resected early-stage HBVassociated $\mathrm{HCC}$.

Abbreviations: AFP, alpha-fetoprotein; HBV-associated HCC, hepatitis B virus-associated hepatocellular carcinoma; OS, overall survival; TACE, transarterial chemoembolization; VCAN, versican. 
patients with early-stage HBV-associated HCC are shown in Table 5. In brief, compared with patients with Haplo1, Haplo2, or Haplo3 and a small tumor size $(\leq 5 \mathrm{~cm})$, those with Haplo4 and a large tumor size $(>5 \mathrm{~cm})$ had a significantly increased risk of death (adjusted HR $=3.00,95 \% \mathrm{CI}$ : $1.67-5.36, p<0.001$, Table 5; Figure 3) after adjusting for tumor size, tumor capsule, and regional invasion (excluding the stratified factor in each stratum). Similarly, patients with Haplo4 and an incomplete tumor capsule had a significantly increased risk of death, compared with patients with Haplo1, Haplo2, or Haplo3 and a complete tumor capsule (adjusted HR $=1.99,95 \%$ CI: $1.12-3.55, p=0.020$, Table 5; Figure 3).

\section{Association of VCAN expression with OS of patients with early-stage HBV- associated $\mathrm{HCC}$}

Since these VCAN SNPs could alter the amino acids in VCAN protein, we retrieved data on the Fudan cohort from
GSE14520 in the NCBI GEO database. These 148 cases of early-stage HBV-associated HCC (BCLC stage A) were divided into 2 groups according to the VCAN expression levels in tumor tissues, that is, the high VCAN expression group ( $\mathrm{n}=76)$, in which the VCAN expression levels were above the median value, and a low VCAN expression group $(n=72)$, in which the VCAN expression levels were below the median value. Our data showed that a low VCAN expression was associated with a better overall patient survival than a high VCAN expression ( $p=0.013$ with the log-rank test; Figure 4).

\section{Discussion}

In the current study, we genotyped 5 different SNPs in the $V C A N$ exon region and associated them with the clinicopathological and survival data of patients with resected early-stage HBV-associated HCC (classified as BCLC A and Child-Pugh class A). Our data showed that rs2652098, rs309559, rs188703,

Table 5 Joint-effects analysis of VCAN haplotypes and clinicopathological characteristics from patients with resected early-stage HBVassociated HCC

\begin{tabular}{|c|c|c|c|c|c|}
\hline Group & Variables & $\begin{array}{l}\text { Death/ } \\
\text { total }\end{array}$ & $\begin{array}{l}\text { Crude HR } \\
(95 \% \mathrm{Cl})\end{array}$ & $\begin{array}{l}\text { Adjusted HR } \\
(95 \% \mathrm{Cl})^{\mathrm{a}}\end{array}$ & $\begin{array}{l}\text { p-value } \\
\text { (adjusted) }^{a}\end{array}$ \\
\hline $\mathrm{A}$ & Haplol/Haplo2/Haplo3 + Tumor size $\leq 5 \mathrm{~cm}$ & $23 / 57$ & 1 & 1 & \\
\hline B & Haplo4 + Tumor size $\leq 5 \mathrm{~cm}$ & $8 / 11$ & $2.13(0.95-4.77)$ & $2.23(1.00-5.00)$ & 0.051 \\
\hline C & Haplol/Haplo2/Haplo3 + Tumor size $>5 \mathrm{~cm}$ & $91 / 125$ & $2.19(1.39-3.47)$ & $1.95(1.22-3.11)$ & 0.005 \\
\hline $\mathrm{D}$ & Haplo4 + Tumor size $>5 \mathrm{~cm}$ & $25 / 29$ & $3.55(2.01-6.28)$ & $3.00(1.67-5.36)$ & $<0.001$ \\
\hline a & Haplol/Haplo2/Haplo3 + Complete tumor capsule & $57 / 105$ & 1 & 1 & \\
\hline b & Haplo4 +Complete tumor capsule & $17 / 22$ & $1.95(1.13-3.35)$ & $1.91(1.11-3.30)$ & 0.02 \\
\hline c & HaploI/Haplo2/Haplo3 + Incomplete tumor capsule & $57 / 77$ & $1.78(1.22-2.59)$ & $1.36(0.92-2.03)$ & 0.12 \\
\hline$d$ & Haplo4 +Incomplete tumor capsule & $16 / 18$ & $2.6 \mathrm{I}(\mathrm{I} .49-4.58)$ & $1.99(1.12-3.55)$ & 0.02 \\
\hline
\end{tabular}

Notes: ${ }^{2}$ Adjusted for tumor size, tumor capsule, regional invasion (excluding the stratified factor in each stratum). Haplotype analysis included rs2652098, rs309559, rs 188703, rs 160278, and rs 160277 SNPs. These $P$-values $<0.05$ were highlighted in bold font.

Abbreviations: $\mathrm{HBV}$-associated HCC, hepatitis B virus-associated hepatocellular carcinoma; HR, hazard ratio; VCAN, versican.

A

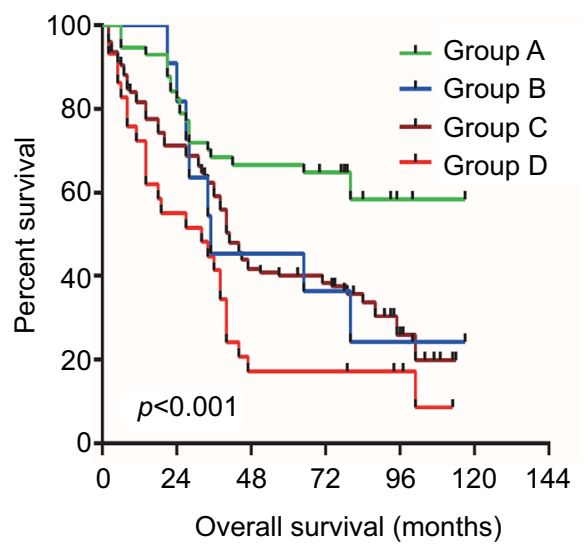

B

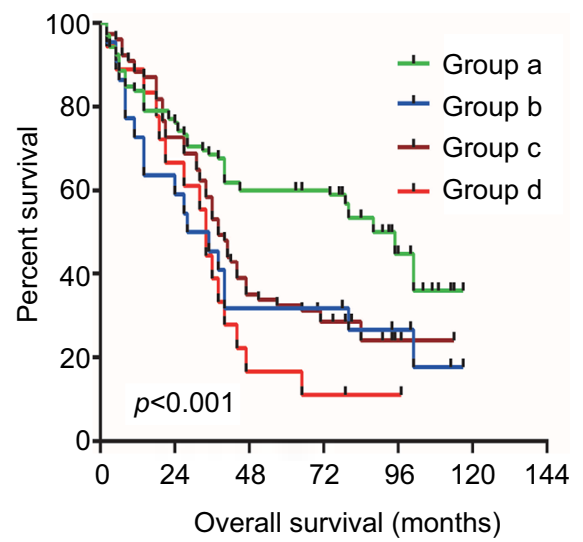

Figure 3 Kaplan-Meier curve of joint-effects analysis in HBV-associated HCC.

Notes: (A) OS stratified by VCAN haplotypes and tumor size cut-off by $5 \mathrm{~cm}$; (B) OS stratified by VCAN haplotypes and tumor capsule complete or not.

Abbreviations: HBV, hepatitis B virus; $\mathrm{HCC}$, hepatocellular carcinoma; OS, overall survival; VCAN, versican. 


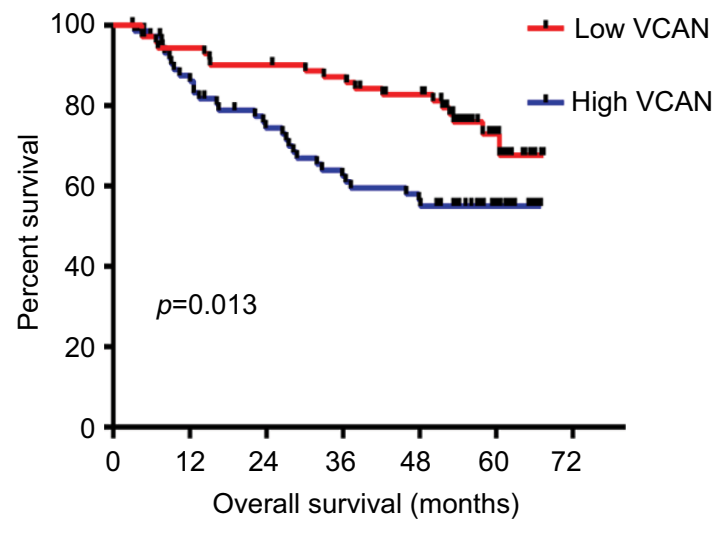

Figure 4 Kaplan-Meier curve prediction of poor OS of patients with resected early-stage $\mathrm{HBV}$-associated HCC stratified by high VCAN expression.

Abbreviations: $\mathrm{HBV}$-associated $\mathrm{HCC}$, hepatitis B virus-associated hepatocellular carcinoma; OS, overall survival; VCAN, versican.

rs160278, and rs160277 were significantly associated with the clinicopathological and survival data of these patients. These 5 SNPs had strong linkage disequilibrium; however, compared with the other 4 VCAN SNPs, rs2652098 was more representative as a potential biomarker for OS of these patients after adjusting for the clinicopathological features. Moreover, haplotype analysis of the association between these 5 SNPs and overall patient survival showed that patients with the TAGTG haplotype had the worst prognosis. In addition, our online data mining analysis showed that a low VCAN expression in HCC tissues was associated with a better OS of HCC patients. Clinicopathological features, like tumor size, tumor capsule, and regional invasion, were also associated with the OS of these patients. Further studies will confirm the usefulness of these $5 V C A N$ SNPs in predicting the prognosis of resected HCC.

In the current study, we first retrieved SNP data on $V C A N$ exonic polymorphisms with amino acid changes from the NCBI dbSNP database (GRCh37.p13) and found rs2652098 (C>T, Ser300Leu), rs309559 (A>G, Lys1516Arg), rs188703 (G>A, Arg1826His), rs160278 (T>A, Phe2301Tyr), and rs160277 (G>T, Asp2937Tyr) for genotyping. To date, there are no reports on these VCAN SNPs being associated with HCC outcome. Our current study demonstrated that these $V C A N$ exonic polymorphisms were associated with the clinicopathological and survival data of patients with resected early-stage HBV-associated HCC. Haplo4 TAGTG (constituted by rs2652098T, rs309559A, rs188703G, rs160278T, and rs $160277 \mathrm{G}$ ) was associated with a poorer prognosis than those with the most common genotype CGAAT. A previous study has revealed that rs $188703 \mathrm{G}$ and $\mathrm{rs} 160277 \mathrm{G}$ can increase the susceptibility to intestinal-type gastric cancer. ${ }^{24}$ rs 188703 , rs160278, and rs160277 are localized on exon 8 of the $V C A N$ gene that codes the GAG-beta domain (G3 domain) of VCAN protein. The GAG-beta domain is associated with enhanced cell proliferation and activation of the epidermal growth factor receptor (EGFR) signaling pathway; ${ }^{9}$ thus, blockade of the GAG-beta domain activity can reduce cell proliferation and EGFR pathway activation as well as reduce susceptibility to cancer development. ${ }^{9}$ Thus, several studies have indicated that targeting VCAN protein expression or activity could be used as a potential tumor therapeutic strategy. ${ }^{10,32-34}$ For example, VCAN G3 has been shown to enhance cancer cell resistance to chemotherapeutic agents, but a selective EGFR inhibitor has been demonstrated to inverse its resistance. ${ }^{10,11,32,35}$ Furthermore, VCAN G3 is able to enhance tumor angiogenesis by formation of a complex together with vascular endothelial growth factor via directly binding to fibronectin. ${ }^{11}$

Our current study also showed that HCC patients with Haplo4 and a larger tumor size or an incomplete tumor capsule had a poorer OS than those with Haplo1, Haplo2, or Haplo3 and a smaller tumor size or a complete tumor capsule. A previous study using PolyPhen prediction (Polymorphism Phenotyping at http://genetics.bwh.harvard.edu/pph/) has shown that rs160277 can affect the function or structure of VCAN protein through transcriptional regulation. ${ }^{36}$ Meanwhile, both rs188703 and rs160277 can affect the function or structure of the GAG-beta domain to interfere with cell proliferation and activation of the EGFR pathway. Overall, the use of these VCAN genetic polymorphisms combined with clinical characteristics might be better at predicting the survival of these early-stage HBV-associated HCC patients after a hepatectomy. However, VCAN, a large chondroitin sulfate proteoglycan, is a major component of the extracellular matrix. ${ }^{37,38}$ Expression of VCAN protein is associated with cell growth and differentiation; ${ }^{39}$ in addition, it is associated with invasion and metastasis of different tumors. ${ }^{11-14,40-45}$ VCAN also plays an important role in transforming growth factor (TGF)- $\beta$-mediated tumor cell invasion and metastasis. ${ }^{46,47}$ TGF- $\beta$-induced VCAN expression is able to activate the nuclear factor- $\mathrm{KB}$ signaling pathway and upregulate the expression of CD44, matrix metalloproteinase 9, and the hyaluronan-mediated motility receptor in ovarian cancer. ${ }^{47}$ VCAN G3 has been shown to promote growth, migration, and metastasis of mouse mammary tumor cells through the upregulation of EGFR signaling. ${ }^{48}$ Indeed, detection of VCAN expression can predict tumor metastasis and progression. ${ }^{49,50}$ Our current data also showed that increased VCAN expression was associated with a poorer OS of HCC patients. Recently, Xia et al found that FoxQ1 promotes HCC metastasis by transactivation of VCAN expression. ${ }^{51}$ In addition, they also demonstrated that VCAN expression 
contributes to a poorer overall patient survival and higher recurrence rates of HCC. ${ }^{51}$ Similarly, human cervical cancer patients with positive VCAN expression had lower OS rates. ${ }^{15,52}$ Thus, further studies will confirm whether detection of VCAN SNPs or protein expression is more useful in predicting the survival of $\mathrm{HCC}$ patients.

Of note, our study does have potential limitations. First, as an SNP study, the sample size was small and a fraction of the clinical data was missing. Second, there was a lack of functional characterizations between these SNPs and VCAN expression or activity.

\section{Conclusion}

Our current study provided a proof-of-principle to associate $V C A N$ rs2652098, rs309559, rs188703, rs160278, and rs160277 SNPs with a poor OS of patients with resected early-stage $\mathrm{HBV}$-associated $\mathrm{HCC}$, even after adjusting for other clinicopathological features, such as tumor size, tumor capsule, or regional invasion. Our current data also confirmed that a low VCAN expression was associated with a better OS of HCC patients than a high VCAN expression. However, future studies are needed to validate our current findings and clarify the underlying mechanisms.

\section{Acknowledgments}

This work was supported in part by the National Nature Science Foundation of China (81560535, 81072321, 30760243 , 30460143, and 30560133), 2009 Program for New Century Excellent Talents in University (NCET), Guangxi Nature Sciences Foundation (GuiKeGong 1104003A-7), and Guangxi Health Ministry Medicine Grant (Key-Scientific Research-Grant Z201018). The present study is also partly supported by Scientific Research Fund of the Health and Family Planning Commission of Guangxi Zhuang Autonomous Region (Z2016318). We would also acknowledge the support provided by the National Key Clinical Specialty Programs (General Surgery and Oncology) and the Key Laboratory of Early Prevention and Treatment for Regional High-IncidenceTumor (Guangxi Medical University), Ministry of Education, China. The authors thank Minhao Peng, Lequn Li, Xiao Qin, Kaiyin Xiao, Xigang Chen, Bin Chen, Zhixiong Su, Ming Su, Zhang Wen, Jingning Lu, Ning Peng, Hai Zhu for providing part of HCC samples for this study, who are from the Department of Hepatobiliary Surgery, the First Affiliated Hospital of Guangxi Medical University. Thanks also go to Xue Qin (Department of Clinical Laboratory, The First Affiliated Hospital of Guangxi Medical University) for providing patient's serum AFP assay results. We would like to acknowledge the researcher Jiaquan Li and Ying Gui from Guangxi Medical University for their contribution to specimen management. The authors thank the contributors of GSE14520 (https://www.ncbi.nlm.nih.gov/geo/query/ acc. $\operatorname{cgi}$ ?acc $=$ GSE14520) for sharing the HCC data on open access. The authors also want to thank the Mingyi Li, Nianping Chen, Ming Chen, Xiaoyu Tan, Huilai Miao, Dong Dai, Ai Mo, Wei Dai, Hao Xu, Yongguang Yang, Manzhou Lin, Guohua Liu, who are from the Departments of Hepatobiliary Surgery, Affiliated Hospital of Guangdong Medical University, Zhanjiang, Guangdong 524001, China, for their contribution to manuscript revision. In addition, we would also like to acknowledge the helpful comments on this paper received from our reviewers.

\section{Disclosure}

The authors report no conflicts of interest in this work.

\section{References}

1. Torre LA, Bray F, Siegel RL, Ferlay J, Lortet-Tieulent J, Jemal A. Global cancer statistics, 2012. CA Cancer J Clin. 2015;65(2):87-108.

2. El-Serag HB, Kanwal F. Epidemiology of hepatocellular carcinoma in the United States: where are we? Where do we go? Hepatology. 2014;60(5):1767-1775.

3. El-Serag HB, Rudolph KL. Hepatocellular carcinoma: epidemiology and molecular carcinogenesis. Gastroenterology. 2007;132(7):2557-2576.

4. Chen W, Zheng R, Baade PD, et al. Cancer statistics in China, 2015. CA Cancer J Clin. 2016;66(2):115-132.

5. Dhir M, Melin AA, Douaiher J, et al. A Review and Update of Treatment Options and Controversies in the Management of Hepatocellular Carcinoma. Ann Surg. 2016;263(6):1112-1125.

6. Forner A, Llovet JM, Bruix J. Hepatocellular carcinoma. Lancet. 2012;379(9822):1245-1255.

7. Pinato DJ, North BV, Sharma R. A novel, externally validated inflammation-based prognostic algorithm in hepatocellular carcinoma: the prognostic nutritional index (PNI). Br J Cancer. 2012;106(8):1439-1445.

8. Iozzo RV, Naso MF, Cannizzaro LA, Wasmuth JJ, McPherson JD. Mapping of the versican proteoglycan gene (CSPG2) to the long arm of human chromosome 5 (5q12-5q14). Genomics. 1992;14(4):845-851.

9. Sheng W, Wang G, Wang Y, et al. The roles of versican V1 and V2 isoforms in cell proliferation and apoptosis. Mol Biol Cell. 2005;16(3): 1330-1340.

10. Du WW, Yang BB, Yang BL, et al. Versican G3 domain modulates breast cancer cell apoptosis: a mechanism for breast cancer cell response to chemotherapy and EGFR therapy. PloS one. 2011;6(11):e26396.

11. Zheng PS, Wen J, Ang LC, et al. Versican/PG-M G3 domain promotes tumor growth and angiogenesis. FASEB J. 2004;18(6):754-756.

12. Cattaruzza S, Schiappacassi M, Kimata K, Colombatti A, Perris R. The globular domains of PG-M/versican modulate the proliferationapoptosis equilibrium and invasive capabilities of tumor cells. FASEB J. 2004;18(6):779-781.

13. Arslan F, Bosserhoff AK, Nickl-Jockschat T, Doerfelt A, Bogdahn $\mathrm{U}$, Hau P. The role of versican isoforms V0/V1 in glioma migration mediated by transforming growth factor-beta2. $\mathrm{Br} J$ Cancer. 2007;96(10):1560-1568.

14. Yee AJ, Akens M, Yang BL, et al. The effect of versican G3 domain on local breast cancer invasiveness and bony metastasis. Breast Cancer Res. 2007;9(4):R47.

15. Kodama J, Hasengaowa, Kusumoto T, et al. Versican expression in human cervical cancer. Eur J Cancer. 2007;43(9):1460-1466. 
16. Zako M, Shinomura T, Ujita M, Ito K, Kimata K. Expression of PG$\mathrm{M}(\mathrm{V} 3)$, an alternatively spliced form of PG-M without a chondroitin sulfate attachment in region in mouse and human tissues. J Biol Chem. 1995;270(8):3914-3918.

17. Dours-Zimmermann MT, Zimmermann DR. A novel glycosaminoglycan attachment domain identified in two alternative splice variants of human versican. J Biol Chem. 1994;269(52):32992-32998.

18. Ito K, Shinomura T, Zako M, Ujita M, Kimata K. Multiple forms of mouse PG-M, a large chondroitin sulfate proteoglycan generated by alternative splicing. J Biol Chem. 1995;270(2):958-965.

19. Kim NS, Lee HH, Jung CK, Jeon HM. Versican expression in tumor epithelial cells is correlated with a good prognosis in gastric cancer. Anticancer Res. 2014;34(10):5613-5619.

20. Voutilainen K, Anttila M, Sillanpaa S, et al. Versican in epithelial ovarian cancer: relation to hyaluronan, clinicopathologic factors and prognosis. Int J Cancer: 2003;107(3):359-364.

21. Suwiwat S, Ricciardelli C, Tammi R, et al. Expression of extracellular matrix components versican, chondroitin sulfate, tenascin, and hyaluronan, and their association with disease outcome in node-negative breast cancer. Clin Cancer Res. 2004;10(7):2491-2498.

22. Ricciardelli C, Mayne K, Sykes PJ, et al. Elevated levels of versican but not decorin predict disease progression in early-stage prostate cancer. Clin Cancer Res. 1998;4(4):963-971.

23. Du WW, Fang L, Yang X, et al. The role of versican in modulating breast cancer cell self-renewal. Mol Cancer Res. 2013;11(5):443-455.

24. Ju H, Lim B, Kim M, et al. Genetic variants A1826H and D2937Y in GAG-beta domain of versican influence susceptibility to intestinal-type gastric cancer. J Cancer Res Clin Oncol. 2010;136(2):195-201.

25. Zimmermann DR, Ruoslahti E. Multiple domains of the large fibroblast proteoglycan, versican. EMBO J. 1989;8(10):2975-2981.

26. Edmondson HA, Steiner PE. Primary carcinoma of the liver: a study of 100 cases among 48,900 necropsies. Cancer. 1954;7(3):462-503.

27. Forner A, Reig ME, de Lope CR, Bruix J. Current strategy for staging and treatment: the BCLC update and future prospects. Semin Liver Dis. 2010;30(1):61-74.

28. Bruix J, Gores GJ, Mazzaferro V. Hepatocellular carcinoma: clinical frontiers and perspectives. Gut. 2014;63(5):844-855.

29. Roessler S, Long EL, Budhu A, et al. Integrative genomic identification of genes on $8 \mathrm{p}$ associated with hepatocellular carcinoma progression and patient survival. Gastroenterology. 2012;142(4):957-966 e912.

30. Roessler S, Jia HL, Budhu A, et al. A unique metastasis gene signature enables prediction of tumor relapse in early-stage hepatocellular carcinoma patients. Cancer Res. 2010;70(24):10202-10212.

31. Gabriel SB, Schaffner SF, Nguyen H, et al. The structure of haplotype blocks in the human genome. Science. 2002;296(5576):2225-2229.

32. Arichi N, Mitsui Y, Hiraki M, et al. Versican is a potential therapeutic target in docetaxel-resistant prostate cancer. Oncoscience. 2015;2(2): 193-204.

33. Wang Z, Li Z, Wang Y, et al. Versican silencing improves the antitumor efficacy of endostatin by alleviating its induced inflammatory and immunosuppressive changes in the tumor microenvironment. Oncol Rep. 2015;33(6):2981-2991.

34. Fujii K, Karpova MB, Asagoe K, Georgiev O, Dummer R, UrosevicMaiwald M. Versican upregulation in Sezary cells alters growth, motility and resistance to chemotherapy. Leukemia. 2015;29(10):2024-2032.
35. Wight TN, Kang I, Merrilees MJ. Versican and the control of inflammation. Matrix Biol. 2014;35:152-161.

36. Ramensky V, Bork P, Sunyaev S. Human non-synonymous SNPs: server and survey. Nucleic Acids Res. 2002;30(17):3894-3900.

37. Doege K, Sasaki M, Yamada Y. Rat and human cartilage proteoglycan (aggrecan) gene structure. Biochem Soc Trans. 1990;18(2): 200-202.

38. Doege KJ, Sasaki M, Kimura T, Yamada Y. Complete coding sequence and deduced primary structure of the human cartilage large aggregating proteoglycan, aggrecan. Human-specific repeats, and additional alternatively spliced forms. J Biol Chem. 1991;266(2):894-902.

39. Naso MF, Zimmermann DR, Iozzo RV. Characterization of the complete genomic structure of the human versican gene and functional analysis of its promoter. J Biol Chem. 1994;269(52):32999-33008.

40. Creighton CJ, Bromberg-White JL, Misek DE, et al. Analysis of tumorhost interactions by gene expression profiling of lung adenocarcinoma xenografts identifies genes involved in tumor formation. Mol Cancer Res. 2005;3(3):119-129.

41. LaPierre DP, Lee DY, Li SZ, et al. The ability of versican to simultaneously cause apoptotic resistance and sensitivity. Cancer Res. 2007;67(10):4742-4750.

42. Ang LC, Zhang Y, Cao L, et al. Versican enhances locomotion of astrocytoma cells and reduces cell adhesion through its G1 domain. J Neuropathol Exp Neurol. 1999;58(6):597-605.

43. Paris S, Sesboue R, Chauzy C, Maingonnat C, Delpech B. Hyaluronectin modulation of lung metastasis in nude mice. Eur $J$ Cancer. 2006;42(18):3253-3259.

44. Ricciardelli C, Russell DL, Ween MP, et al. Formation of hyaluronanand versican-rich pericellular matrix by prostate cancer cells promotes cell motility. J Biol Chem. 2007;282(14):10814-10825.

45. Miquel-Serra L, Serra M, Hernandez D, et al. V3 versican isoform expression has a dual role in human melanoma tumor growth and metastasis. Lab Invest. 2006;86(9):889-901.

46. Li F, Li S, Cheng T. TGF-betal promotes osteosarcoma cell migration and invasion through the miR-143-versican pathway. Cell Physiol Biochem. 2014;34(6):2169-2179.

47. Yeung TL, Leung CS, Wong KK, et al. TGF-beta modulates ovarian cancer invasion by upregulating CAF-derived versican in the tumor microenvironment. Cancer Res. 2013;73(16):5016-5028.

48. Du WW, Yang BB, Shatseva TA, et al. Versican G3 promotes mouse mammary tumor cell growth, migration, and metastasis by influencing EGF receptor signaling. PloS one. 2010;5(11):e13828.

49. Damasceno KA, Bertagnolli AC, Estrela-Lima A, et al. Versican expression in canine carcinomas in benign mixed tumours: is there an association with clinical pathological factors, invasion and overall survival? BMC Vet Res. 2012;8:195.

50. Kischel P, Waltregny D, Dumont B, et al. Versican overexpression in human breast cancer lesions: known and new isoforms for stromal tumor targeting. Int J Cancer. 2010;126(3):640-650.

51. Xia L, Huang W, Tian D, et al. Forkhead box Q1 promotes hepatocellular carcinoma metastasis by transactivating ZEB2 and VersicanV1 expression. Hepatology. 2014;59(3):958-973.

52. Ghosh S, Albitar L, LeBaron R, et al. Up-regulation of stromal versican expression in advanced stage serous ovarian cancer. Gynecol Oncol. 2010;119(1):114-120.
Cancer Management and Research

\section{Publish your work in this journal}

Cancer Management and Research is an international, peer-reviewed open access journal focusing on cancer research and the optimal use of preventative and integrated treatment interventions to achieve improved outcomes, enhanced survival and quality of life for the cancer patient. The manuscript management system is completely online and includes

\section{Dovepress}

a very quick and fair peer-review system, which is all easy to use. Visit $\mathrm{http}: / / \mathrm{www}$.dovepress.com/testimonials.php to read real quotes from published authors. 